\title{
EFEITO DE FONTES E DOSES DE NITROGÊNIO SOBRE O CRESCIMENTO INICIAL E QUALIDADE DE MUDAS DE JACARANDÁ-DA-BAHIA (Dalbergia nigra (Vell.) Fr. All. ex Benth.) ${ }^{1}$
}

Vanderleia Braga Marques², Haroldo Nogueira de Paiva ${ }^{3}$, José Mauro Gomes ${ }^{3}$, Júlio César Lima Neves ${ }^{4}$ e

Daíse Cardoso de Souza Bernardino ${ }^{5}$

\begin{abstract}
RESUMO - O trabalho teve como objetivo avaliar o efeito de fontes e doses de nitrogênio no crescimento inicial e na qualidade de mudas de jacarandá-da-bahia (Dalbergia nigra (Vell.) Fr. All. ex Benth.) produzidas em três tipos de solo (Argissolo Vermelho-Amarelo, Cambissolo e Latossolo Vermelho-Amarelo). As fontes de $\mathrm{N}$ utilizadas foram nitrato de amônio, nitrato de cálcio e sulfato de amônio em cinco doses $(0,50,100$, 150 e $200 \mathrm{mg} / \mathrm{dm}^{3} \mathrm{de} \mathrm{N}$ ) igualmente parceladas e aplicadas aos 25, 50, 75 e 100 dias após a semeadura. O delineamento estatístico adotado foi o inteiramente casualizado, em esquema fatorial (3 fontes de nitrogênio x 5 doses x 3 tipos de solos), com quatro repetições. Os dados foram submetidos à análise de variância, testes de médias e análise de regressão. Aos 125 dias após a semeadura, o melhor padrão de qualidade foi observado nas mudas produzidas no Argissolo Vermelho-Amarelo, tendo como fonte o sulfato de amônio, com dose de $180 \mathrm{mg} / \mathrm{dm}^{3}$ de $\mathrm{N}$.
\end{abstract}

Palavras chave: Jacarandá-da-bahia, qualidade de mudas, fertilização e nitrogênio.

\section{EFFECT OF SOURCES AND DOSES OF NITROGEN ON THE INITIAL GROWTH AND QUALITY OF SEEDLINGS OF JACARANDÁ-DA-BAHIA (Dalbergia nigra (Vell.) Fr. All. ex Benth.).}

\begin{abstract}
The objective of this work was to evaluate the effect of sources and doses of nitrogen on the initial growth and quality of jacarandá-da-Bahia (Dalbergia nigra (Vell.) Fr. All. ex Benth.) seedlings produced in three types of soils (RED YELLOW ARGISOL, CAMBISOL and RED YELLOW LATOSOL). The nitrogen sources were: ammonium nitrate, calcium nitrate and ammonium sulfate, in five doses $(0,50,100,150$ and 200 $\mathrm{mg} / \mathrm{dm}^{3}$ of $\mathrm{N}$ ) equally parceled out and applied on the $25^{\text {th }}, 50^{\text {th }}, 75^{\text {th }}$ and 100 days after sowing. The experiment was arranged in a complete randomized blocks, in ( $3 \times 5 \times 3)$ factorial design, with four repetitions. At 125 days after sowing, the best quality standard was found in seedlings produced in the RED YELLOW ARGISOL, having ammonium sulfate as nitrogen source, with the dose of $180 \mathrm{mg} / \mathrm{dm}^{3}$ of $\mathrm{N}$.
\end{abstract}

Keywords: Jacarandá-da-Bahia, seedling quality, fertilization, nitrogen.

\footnotetext{
${ }^{1}$ Recebido em 12.07.2004 e aceito para publicação em 05.04.2006.

${ }^{2}$ Rua Manacás, 35, 29.260-000 D. Martins-E.S. E-mail: <vanderleiabraga@ yahoo.com.br>.

${ }^{3}$ Departamento de Engenharia Florestal da UFV, 36 571-000 Viçosa-MG. E-mail:<hnpaiva@ufv.br>.

${ }^{4}$ Departamento de Solos da UFV, 36.571-000 Viçosa-MG.

${ }^{5}$ Laboratório de Patologia Florestal e Genética da Interação Planta-Patôgeno - BIOAGRO, UFV.
} 


\section{INTRODUÇÃO}

Jacarandá-da-bahia (Dalbergia nigra (Vell.) Fr. All. Ex Benth. é uma espécie florestal nativa da família Leguminosae-Papilionoidea que ocorre no sul da Bahia e Sudeste do país, cuja madeira é mundialmente conhecida na construção de mobiliário de luxo, sendo também muito promissora em reflorestamentos (LORENZI, 1998).

Os projetos que objetivam a conservação e exploração de espécies florestais nativas dependem da formação de mudas. Assim, a recuperação de áreas, a conservação dos solos, o estabelecimento de bancos de germoplasma, os programas de melhoramento e os plantios comerciais para exploração econômica de frutos, madeira e produtos medicinais são baseados na coleta de sementes e reprodução das espécies (MELO et al., 1998). Portanto, para que essa e outras essências sejam cultivadas, é necessário maior conhecimento sobre a sua silvicultura, incluindo-se a nutrição mineral, com vistas a aperfeiçoar o sistema de produção de mudas com técnicas que promovam melhor qualidade das plantas, resultando em maior potencial de sobrevivência e crescimento após o plantio (CARNEIRO, 1995).

De todos os nutrientes, o nitrogênio é o que se encontra em maiores concentrações nos vegetais superiores. Na grande maioria das espécies cultivadas, principalmente as de ciclo anual, o metabolismo do nitrogênio tem sido bastante estudado (BLEVINS, 1989), não ocorrendo o mesmo para espécies florestais, sobretudo as nativas. Segundo Gonçalves (1995), as características e a quantidade de adubos a serem aplicados dependerão das necessidades nutricionais da espécie utilizada, da fertilidade do solo, da forma de reação dos adubos e de fatores de ordem econômica.

Devido à escassez de informações sobre as exigências nutricionais de espécies florestais nativas quanto à nutrição nitrogenada, neste trabalho objetivouse avaliar o efeito de fontes e doses de nitrogênio no crescimento inicial e qualidade de mudas de jacarandáda-bahia (Dalbergia nigra (Vell.) Fr. All. Ex Benth., produzidas em três tipos de solo.

\section{MATERIAL E MÉTODOS}

O experimento foi instalado e conduzido em casa de vegetação no viveiro de pesquisas em propagação de plantas lenhosas do Departamento de Engenharia Florestal da Universidade Federal de Viçosa em Viçosa, MG, no período de novembro de 2003 a fevereiro de 2004.
As amostras de solos usadas como substrato na produção das mudas foram retiradas cerca de $30 \mathrm{~cm}$ abaixo da camada superficial de três tipos de solos (Argissolo VErmelho-Amarelo, Cambissolo e Latossolo Vermelho-Amarelo) predominantes na região da Zona da Mata de Minas Gerais (RESENDE et al., 1988). Após a secagem ao ar e peneiramento em malha de $5 \mathrm{~mm}$, procedeu-se à correção da acidez dos solos, usando uma mistura de $\mathrm{CaCO}_{3}$ e $\mathrm{MgCO}_{3}$, na relação estequiométrica de $4: 1$. A necessidade de calagem foi calculada com base na análise química dos solos (Quadro 1) e para elevar a saturação por bases a $60 \%$. Após a incorporação do corretivo, o solo foi incubado por 30 dias, com manutenção do teor de umidade à capacidade de campo. Após esse período, os solos receberam adubação básica de macronutrientes por solução, nas seguintes doses: $\mathrm{P}=300 \mathrm{mg} / \mathrm{dm}^{3}, \mathrm{~K}=100 \mathrm{mg} / \mathrm{dm}^{3} \mathrm{e}$ $\mathrm{S}=40 \mathrm{mg} / \mathrm{dm}^{3}$, tendo como fontes $\mathrm{NaH}_{2} \mathrm{PO}_{4} \cdot \mathrm{H}_{2} \mathrm{O}, \mathrm{KCl}$ e $\mathrm{K}_{2} \mathrm{SO}_{4}$, conforme sugerido por Passos (1994), e ainda uma solução de micronutrientes, nas seguintes doses: $\mathrm{B}=0,81 \mathrm{mg} / \mathrm{dm}^{3}\left(\mathrm{H}_{3} \mathrm{BO}_{3}\right), \mathrm{Cu}=1,33 \mathrm{mg} / \mathrm{dm}^{3}\left(\mathrm{CuSO}_{4} \cdot 5 \mathrm{H}_{2} \mathrm{O}\right)$, $\mathrm{Mo}=0,15 \mathrm{mg} / \mathrm{dm}^{3}\left[\left(\mathrm{NH}_{4}\right)_{6} \mathrm{Mo}_{7} \mathrm{O}_{24} \cdot 4 \mathrm{H}_{2} \mathrm{O}\right], \mathrm{Mn}=3,66 \mathrm{mg} /$ $\mathrm{dm}^{3}\left(\mathrm{MnCl}_{2} \cdot \mathrm{H}_{2} \mathrm{O}\right)$ e $\mathrm{Zn}=4,0 \mathrm{mg} / \mathrm{dm}^{3}\left(\mathrm{ZnSO}_{4} \cdot 7 \mathrm{H}_{2} \mathrm{O}\right)$, de acordo com Alvarez V. (1974), e, então, foram acondicionados em vasos com capacidade para 1,5 $\mathrm{dm}^{3}$ de solo.

Os tratamentos foram constituídos por três fontes de nitrogênio aplicadas por solução, na forma de nitrato de amônio $\left[\mathrm{NH}_{4} \mathrm{NO}_{3}\right]$, sulfato de amônio $\left[\left(\mathrm{NH}_{4}\right)_{2} \mathrm{SO}_{4}\right]$ e nitrato de cálcio $\left[\mathrm{Ca}\left(\mathrm{NO}_{3}\right)_{2}\right]$; e cinco doses $(0,50$, 100,150 e $200 \mathrm{mg} / \mathrm{dm}^{3} \mathrm{de} \mathrm{N}$ ), aplicadas em quatro porções iguais, aos 25, 50, 75 e 100 dias após a semeadura. A unidade experimental foi constituída por um vaso com capacidade por $1,5 \mathrm{dm}^{3}$ de solo, contendo uma muda. O delineamento estatístico adotado foi o inteiramente casualizado, em esquema fatorial ( 3 x 5 x 3), correspondendo a três fontes, cinco doses e três solos, com quatro repetições, num total de 180 vasos.

As sementes do jacarandá-da-bahia foram inoculadas com estirpes selecionadas de Bradyrhizobium, fornecidas pelo Centro Nacional de Pesquisa em Agrobiologia/EMBRAPA, Seropédica, RJ. A semeadura foi efetuada manual e diretamente nos vasos, colocando-se 10 sementes/vaso. No raleio, realizado 20 dias após a semeadura, optou-se pela muda mais vigorosa e central. 
Quadro 1 - Análise química dos solos utilizados na produção das mudas Table 1 - Chemical analysis of soils used for seedling production

\begin{tabular}{lccccccccccc}
\hline \multirow{1}{*}{ Solo } & ${ }^{1} \mathrm{pH}$ & ${ }^{2} \mathrm{P}$ & ${ }^{2} \mathrm{~K}$ & ${ }^{3} \mathrm{Ca}^{2+}$ & ${ }^{3} \mathrm{Mg}^{2+}$ & ${ }^{3} \mathrm{Al}^{3+}$ & ${ }^{4} \mathrm{H}+\mathrm{Al}$ & $\mathrm{SB}$ & $(\mathrm{T})$ & $\mathrm{V}(\%)$ & ${ }^{5} \mathrm{MO}$ \\
\cline { 2 - 11 } & $\mathrm{H}_{2} \mathrm{O}$ & $\mathrm{mg} / \mathrm{dm}^{3}$ & & & & & $\mathrm{cmol}_{\mathrm{c}} / \mathrm{dm}^{3}$ & & & $\mathrm{dag} / \mathrm{kg}$ \\
\hline ARGISSOLO & 5,64 & 1,5 & 16 & 1,74 & 0,17 & 0,00 & 3,0 & 1,95 & 4,95 & 39,4 & 2,82 \\
CAMBISSOLO & 5,60 & 1,5 & 66 & 1,00 & 0,31 & 0,00 & 1,7 & 1,48 & 3,18 & 46,5 & 2,55 \\
LATOSSOLO & 5,40 & 2,5 & 26 & 0,17 & 0,09 & 0,00 & 2,0 & 0,33 & 2,33 & 14,2 & 2,69 \\
\hline
\end{tabular}

${ }^{1} \mathrm{pH}$ em água - Relação 1: 2,5. ${ }^{2} \mathrm{Pe} \mathrm{K}$ - Extrator Mehlich $1 .{ }^{3} \mathrm{Ca}^{2+}, \mathrm{Mg}^{2+} \mathrm{e} \mathrm{Al}{ }^{3+}$ - Extrator: $\mathrm{KCl} 1 \mathrm{~mol} / \mathrm{L} .{ }^{4} \mathrm{H}+\mathrm{Al}-$ Extrator Acetato de Cálcio 0,5 mol/L $-\mathrm{pH} 7,0 .{ }^{5} \mathrm{MO}=\mathrm{C}$. Org x 1,724 - Método Walkley-Black.

As características morfológicas e suas relações para determinação dos índices de qualidade das mudas, segundo Gomes (2001), foram selecionadas na colheita do experimento, 125 dias após a semeadura, quando também se verificram a presença e o número de nódulos no sistema radicular. Essas características foram a altura da parte aérea $(\mathrm{H})$, o diâmetro do coleto (DC), o peso de matéria seca da parte aérea (PMSPA), o peso de matéria seca das raízes (PMSR), o peso de matéria seca total (PMST), a relação altura da parte aérea/diâmetro do coleto (RHDC), a relação altura da parte aérea/peso de matéria seca da parte aérea (RHPMSPA), a relação peso de matéria seca da parte aérea/peso de matéria seca das raízes (RPMSPAR) e o índice de qualidade de Dickson (IQD), mediante a fórmula:

$$
\mathrm{IQD}=\frac{\operatorname{PMST}(\mathrm{g})}{H(\mathrm{~cm}) / D C(\mathrm{~mm})+\operatorname{PMSPA}(\mathrm{g}) / \operatorname{PMSR}(\mathrm{g})}
$$

Os dados foram interpretados estatisticamente por meio de análise de variância (Quadro 2), testes de médias e análise de regressão, utilizando-se o software Sistema de Análises Estatística e Genética - SAEG
(EUCLYDES, 1997). Na escolha das equações de regressão, consideraram-se a significância dos coeficientes, testada até o nível de $15 \%$ de probabilidade, e o coeficiente de determinação $\left(\mathrm{R}^{2}\right)$.

\section{RESULTADOS E DISCUSSÃO}

Verificou-se que a altura da parte aérea $(\mathrm{H})$ foi significativamente afetada pelas doses de $\mathrm{N}$ em cada solo, aumentando linearmente, para Argissolo VermelhoAmarelo e Cambissolo, com o acréscimo das doses, independentemente da fonte aplicada (Figura 1). No Latossolo Vermelho-Amarelo, o efeito quadrático possibilitou a determinação do maior valor para a $\mathrm{H}$ das mudas $(54,3 \mathrm{~cm})$, obtido na dose de $140 \mathrm{mg} / \mathrm{dm}^{3}$ de N. As plantas referentes ao controle (dose $=0 \mathrm{mg}$ / $\mathrm{dm}^{3}$ ) apresentaram crescimento em altura inferior a 50\%, comparativamente aos melhores tratamentos. Sobre a altura, também se verificou o efeito principal de fonte, sendo maiores médias obtidas com as fontes amoniacais, em que o sulfato de amônio superou em quase duas vezes a média obtida com o nitrato de cálcio (Figura 1B).

Quadro 2 - Resumo da análise de variância das características e relações estudadas de mudas de jacarandá-da-bahia, avaliadas aos 125 dias após a semeadura

Table 2 - Summary of analysis of variance for the studied characteristics and relationships of jacarandá-da-bahia seedlings, evaluated at 125 days after sowing

\begin{tabular}{|c|c|c|c|c|c|c|c|c|c|c|}
\hline \multirow[t]{2}{*}{$\mathrm{FV}$} & \multirow[t]{2}{*}{ GL } & \multicolumn{9}{|c|}{ QUADRADO MÉDIO } \\
\hline & & $\mathrm{H}$ & $\mathrm{DC}$ & RHDC & PMST & PMSPA & PMSR & RHPMSPA & RPMSPAR & IQD \\
\hline Solo (S) & 2 & $1728,63 *$ & $35,79 *$ & $12,21^{\mathrm{ns}}$ & $74,53 *$ & $37,12 *$ & $6,54 *$ & $7364,91 *$ & $0,57 \mathrm{~ns}$ & $0,59 *$ \\
\hline Fonte (F) & 2 & $4121,76^{*}$ & $13,10^{*}$ & $19,35 *$ & $66,45^{*}$ & $37,40 *$ & $4,16^{*}$ & $808,76^{\mathrm{ns}}$ & $0,60^{\mathrm{ns}}$ & $0,20 *$ \\
\hline Dose (D) & 4 & $7438,79 *$ & $25,36^{*}$ & $109,95 *$ & $86,81 *$ & $54,47 *$ & $3,89 *$ & $12648,10 *$ & $21,47 *$ & $0,32 *$ \\
\hline$S \times F$ & 4 & $321,19^{\mathrm{ns}}$ & $0,15^{\mathrm{ns}}$ & $13,47^{\mathrm{ns}}$ & $0,63^{\mathrm{ns}}$ & $0,40 \mathrm{~ns}$ & $0,19^{\mathrm{ns}}$ & $169,52^{\mathrm{ns}}$ & $2,75^{*}$ & $0,002^{\mathrm{ns}}$ \\
\hline$S \times D$ & 8 & $506,42 *$ & $2,56^{*}$ & $11,30^{\mathrm{ns}}$ & $10,20 *$ & $6,20 *$ & $0,54 *$ & $3360,25 *$ & $1,52 \mathrm{~ns}$ & $0,06^{*}$ \\
\hline $\mathrm{F} \times \mathrm{D}$ & 8 & $445,07^{\mathrm{ns}}$ & $2,42 *$ & $14,44 *$ & $8,98 *$ & $5,36^{*}$ & $0,57 *$ & $197,84^{\mathrm{ns}}$ & $1,05^{\mathrm{ns}}$ & $0,04 *$ \\
\hline$S \times F \times D$ & 16 & $374,42 \mathrm{~ns}$ & $1,21^{\mathrm{ns}}$ & $4,64^{\mathrm{ns}}$ & $5,47^{\mathrm{ns}}$ & $3,41^{\mathrm{ns}}$ & $0,33^{\mathrm{ns}}$ & $87,79^{\mathrm{ns}}$ & $1,46^{\mathrm{ns}}$ & 0,02 ns \\
\hline Resíduo & 135 & 240,33 & 1,02 & 5,71 & 3,72 & 2,17 & 0,26 & 293,46 & 1,02 & 0,016 \\
\hline $\mathrm{CV}(\%)$ & & 34,96 & 23,76 & 23,72 & 63,96 & 67,65 & 62,11 & 47,88 & 39,59 & 57,33 \\
\hline
\end{tabular}

* Significativo a $5 \%$ de probabilidade, pelo teste F. ${ }^{\text {ns }}$ Não-significativo a $5 \%$ de probabilidade, pelo teste F.

* Significant at $5 \%$ of probability, for test $F .{ }^{n s}$ Not significant at $5 \%$ of probability, for test $F$. 

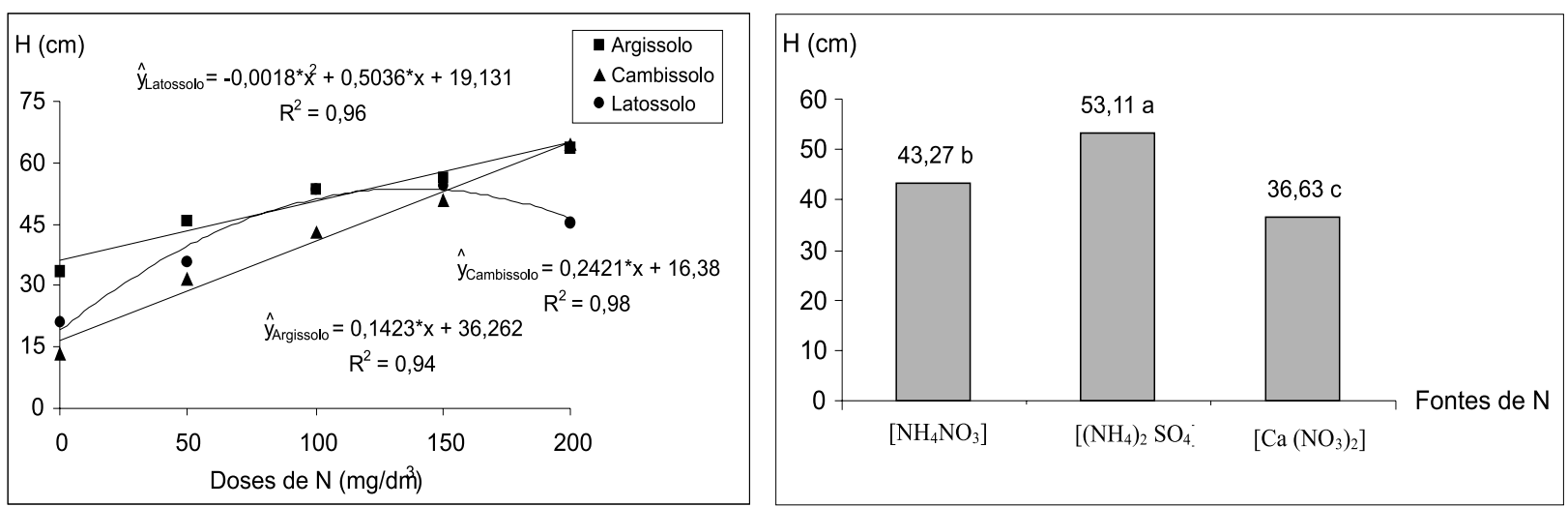

Figura 1A - Altura da parte aérea $(\mathrm{H})$ das mudas de jacarandá-da-bahia, aos 125 dias, em resposta às doses de $\mathrm{N}$, nos três solos estudados. * Coeficientes significativos a 5\%.

Figura 1B - Altura da parte aérea $(\mathrm{H})$ das mudas de jacarandá-da-bahia em resposta às fontes de $\mathrm{N}$ aplicadas. Médias seguidas de uma mesma letra não diferem entre si pelo teste de Tukey a $5 \%$.

Figure $1 A$ - Shoot height $(H)$ of jacarandá-da-bahia seedlings as a function of $N$ doses, for three studied soils. * significant coefficients at $5 \%$.

Figure $1 B$ - Shoot height $(H)$ of jacarandá-da-bahia seedlings as a function of $N$ sources. Means followed by the same letter are not significantly different at 5\% probability level (Tukey's test).

Apesar de a altura ser um excelente parâmetro para avaliar o padrão de qualidade das mudas, alguns trabalhos apontaram resultados contraditórios, como o de Barros et al. (1978), em que os autores constataram, em mudas de Eucalyptus grandis com as maiores alturas, menores taxas de crescimento e de sobrevivência após o plantio.

Sobre o diâmetro do coleto (DC) das mudas, verificou-se efeito significativo nas interações solo $\mathrm{x}$ dose e fonte $\mathrm{x}$ dose. Dentre os solos, observou-se no Cambissolo que os valores dessa característica aumentaram linearmente com as doses, enquanto no Argissolo Vermelho-Amarelo e Latossolo VermelhoAmarelo os maiores valores obtidos foram de 5,81 e $4,39 \mathrm{~mm}$, nas doses de 151 e $126 \mathrm{mg} / \mathrm{dm}^{3} \mathrm{de} \mathrm{N}$, respectivamente, detectados por meio de modelos quadráticos (Figura 2A). Na interação fonte $\mathrm{x}$ dose, verificou-se efeito quadrático sobre o DC nas três fontes aplicadas, com a existência de pontos de máxima produção, 5,0; 5,6; e 4,22 mm, respectivamente, para nitrato de amônio, sulfato de amônio e nitrato de cálcio em torno das doses de 175, 174 e $128 \mathrm{mg} / \mathrm{dm}^{3} \mathrm{de} \mathrm{N}$, respectivamente (Figura $2 \mathrm{~B}$ ).

Os resultados obtidos para a relação altura da parte aérea/diâmetro do coleto (RHDC) aumentaram linearmente com as doses aplicadas (Figura 3). Contudo, segundo Carneiro (1983), quanto menor for esse valor maior será a capacidade das mudas em sobreviver e estabelecer-se. Possivelmente, nesse estudo o maior crescimento em altura das mudas, elevando-se o valor da relação, ocorreu em conseqüência da adubação nitrogenada que favoreceu o crescimento vegetativo. Segundo Sturion e Antunes (2000), a relação altura da parte aérea/ diâmetro do coleto (RHDC) constitui uma das características usadas para avaliar a qualidade de mudas florestais, pois, além de refletir o acúmulo de reservas, assegura maior resistência e melhor fixação no solo. Para Moreira e Moreira (1996), essa variável é reconhecida como um dos melhores, senão o melhor indicador do padrão de qualidade de mudas, sendo, em geral, o mais indicado para determinar a capacidade de sobrevivência de mudas no campo (DANIEL et al., 1997; CARNEIRO, 1995).

O peso de matéria seca da parte aérea (PMSPA) das mudas foi significativamente afetado pela interação solo x dose de nitrogênio. No Cambissolo, a produção foi crescente e linearmente relacionada com a dose, variando de 0,42 a 3,72 g, quando a dose aplicada variou de zero (controle) a $200 \mathrm{mg} / \mathrm{dm}^{3} \mathrm{de} \mathrm{N}$, respectivamente. Nos outros solos, o efeito quadrático sobre o PMSPA permitiu ajustar equações para estimar a dose associada com a produção máxima de 4,5 g para Argissolo VermelhoAmarelo e 2,7 g para Latossolo Vermelho-Amarelo, que foram da ordem de 196 e $158 \mathrm{mg} / \mathrm{dm}^{3} \mathrm{de} \mathrm{N}$, respectivamente (Figura 4A). A grande alocação de matéria seca para 
a parte aérea ocorre, geralmente, na maioria das plantas com suprimento adequado de nutrientes (SCHUMACHER et al., 2004). Possivelmente por este motivo o Argissolo Vermelho-Amarelo, solo naturalmente mais fértil que, somado com a adubação aplicada, tenha possibilitado o maior valor para essa e outras características. $\mathrm{Na}$ interação fonte $\mathrm{x}$ dose, $\mathrm{o}$ efeito do $\mathrm{N}$-amoniacal foi linear, variando de 0,7 a 5,2 g para o sulfato de amônio e 0,4 a 3,6 g para o nitrato de amônio, nas doses de 0 a $200 \mathrm{mg} / \mathrm{dm}^{3}$ de $\mathrm{N}$, respectivamente, enquanto na forma nítrica o efeito foi quadrático, verificando-se o maior valor, $1,98 \mathrm{~g}$, na dose de $140 \mathrm{mg} / \mathrm{dm}^{3} \mathrm{de} \mathrm{N}$, ou seja, duas vezes menos que o encontrado com o sulfato de amônio na maior dose aplicada (Figura 4B). Resultados semelhantes foram encontrados por Locatelii et al. (1984), em que o sulfato de amônio proporcionou a máxima produção de matéria seca da parte aérea em mudas de eucalipto.

O peso de matéria seca das raízes (PMSR), à semelhança do PMSPA, também foi significativamente afetado pela interação solo x dose de nitrogênio. Constatou-se efeito linear no Cambissolo, com produção de 1,15 g na maior dose usada. Nos outros solos, o efeito quadrático possibilitou a determinação dos pontos de máxima produção, 1,60 g para Argissolo VermelhoAmarelo e 0,83 g para Latossolo Vermelho-Amarelo, obtidos nas doses de 140 e $121 \mathrm{mg} / \mathrm{dm}^{3}$ de $\mathrm{N}$,

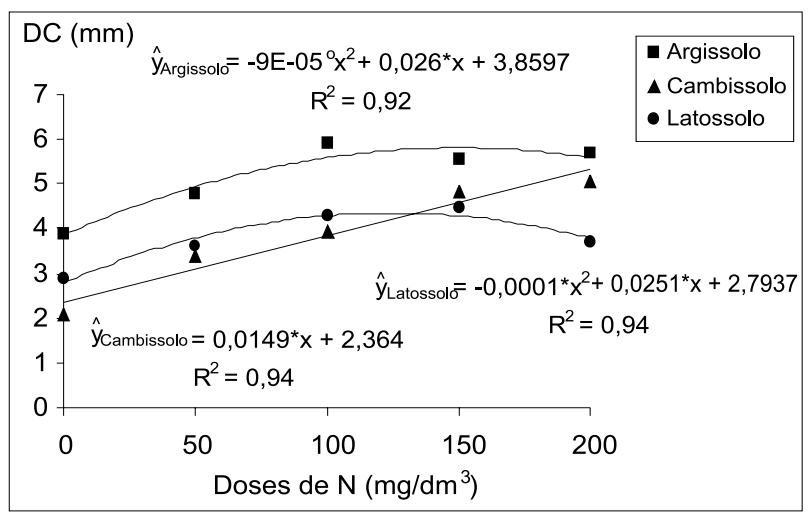

respectivamente (Figura 5A). A maior produção de raízes, à semelhança da maior produção de biomassa da parte aérea, novamente no Argissolo VermelhoAmarelo, além de estar relacionada com as melhores condições químicas desse solo, também pode ser associada, de acordo com Gonçalves et al. (2000), às características físicas dos solos tropicais e subtropicais, em que o elevado grau de agregação faz que a permeabilidade e o potencial de lixiviação das bases dos solos sejam muito elevados, o que pode ser atribuído em menor grau ao Argissolo Vermelho-Amarelo, quando comparado com os outros dois solos testados. $\mathrm{Na}$ interação fonte $\mathrm{x}$ dose, as três fontes aplicadas apresentaram comportamento quadrático sobre essa característica, verificando-se maior produção com as fontes amoniacais. Nas doses de 186 e $172 \mathrm{mg} / \mathrm{dm}^{3}$ de sulfato de amônio e nitrato de amônio, respectivamente, o PMSR foi de 1,68 e 1,14 g, enquanto na dose de 133 $\mathrm{mg} / \mathrm{dm}^{3}$ de nitrato de cálcio foi de $0,68 \mathrm{~g}$, ou seja, produção duas vezes menor que o obtido com sulfato de amônio (Figura 5B). Krajina et al. (1973) reportaram, em experimento com coníferas sob o efeito da adubação nitrogenada, que para Pinus contorta o suprimento com a fonte amoniacal ocasionou resultados significativamente maiores em termos de peso de matéria seca e área foliar total do que o observado com a fonte nítrica.

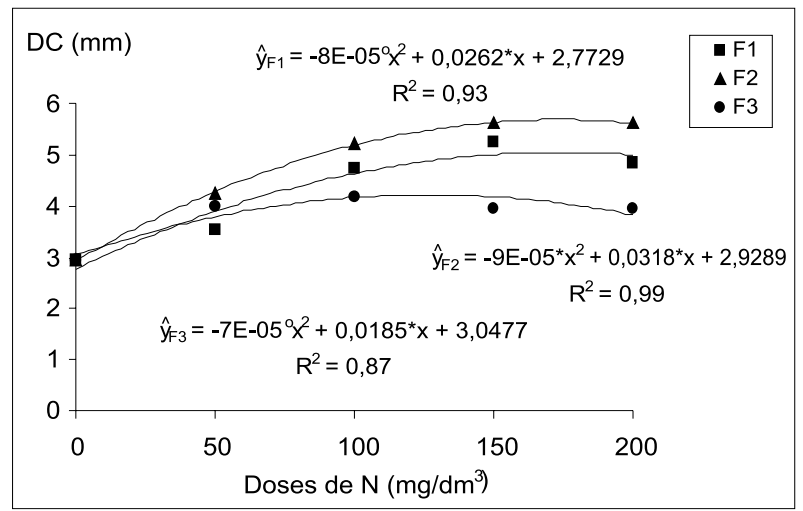

Figura 2A - Diâmetro do coleto (DC) das mudas de jacarandá-da-bahia em resposta às doses de N, nos três solos estudados. $* \mathrm{e}^{\circ}$ coeficientes significativos a 5 e $10 \%$, respectivamente.

Figura 2B - Diâmetro do coleto (DC) das mudas de jacarandá-da-bahia em resposta às doses de N, nas três fontes nitrogenadas $(\mathrm{F} 1=$ nitrato de amônio; $\mathrm{F} 2$ = sulfato de amônio e F3 = nitrato de cálcio $) .{ }^{*} \mathrm{e}^{\circ}$ coeficientes significativos a 5 e $10 \%$, respectivamente.

Figure 2A - Basal diameter (DC) of jacarandá-da-bahia seedlings as a function of $N$ doses, for three studied soils. * and ${ }^{\circ}$, significant coefficients at 5 and $10 \%$, respectively.

Figure 2B - Basal diameter (DC) of jacarandá-da-bahia seedlings as a function of $N$ doses, for three nitrogen sources $(F 1=$ ammonium nitrate; $F 2=$ ammonium sulfate and $F 3=$ calcium nitrate $) .^{*}$ and $^{\circ}$, significant coefficients at 5 and $10 \%$, respectively. 


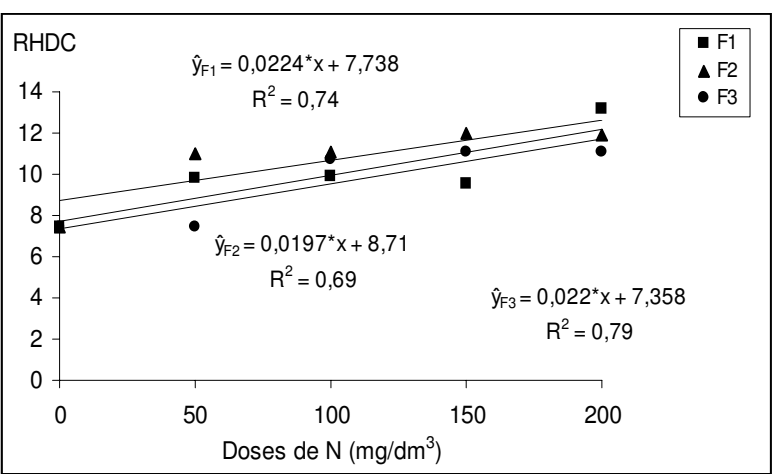

Figura 3-Relação altura da parte aérea/diâmetro do coleto (RHDC) de mudas de jacarandá-da-bahia em resposta às doses de $\mathrm{N}$ aplicadas, nas três fontes nitrogenadas $(\mathrm{F} 1=$ nitrato de amônio, $\mathrm{F} 2$ = sulfato de amônio e F3 = nitrato de cálcio). * coeficientes significativos a $5 \%$.

Figure 3-Shoot height/basal diameter ratio(RHDC) ofjacarandáda-bahia seedlings as a function of $N$ doses, for three nitrogen $\operatorname{sources}(F 1=$ ammonium nitrate; $F 2=$ ammonium sulfate and $\mathrm{F} 3=$ calcium nitrate). $*$ significant coefficients at $5 \%$.

Na relação peso de matéria seca da parte aérea/peso de matéria seca das raízes (RPMSPAR), apesar da análise de variância mostrar efeito significativo na interação solo $\mathrm{x}$ fonte, verificou-se, após o desdobramento por meio de teste de médias, que não houve diferença significativa entre as fontes de $\mathrm{N}$ em cada solo estudado. No entanto, verificou-se que essa relação aumentou linearmente com a elavação das doses (Figura 6). Segundo Parviainen (1981), essa relação é considerada confiável e eficiente para expressar a qualidade das mudas. Sem definir a espécie, Brissette (1984) propôs 2,0 como a melhor relação entre essas características, havendo, porém, outros valores na literatura que variam com a espécie, o sítio e outras características, como os valores encontrados para Pinus taeda e Tabebuia impetiginosa, por Boyer e South (1987) e Cruz et al. (2004), respectivamente.

Para o peso de matéria seca total (PMST), observouse na interação solo x dose, à semelhança do PMSPA e PMSR, efeito quadrático, nos quais o maior valor (6,05 g) foi obtido no Argissolo Vermelho-Amarelo, na dose de $180 \mathrm{mg} / \mathrm{dm}^{3}$ de $\mathrm{N}$, e o menor $(3,19 \mathrm{~g})$ no Latossolo Vermelho-Amarelo, na dose de $128 \mathrm{mg} / \mathrm{dm}^{3} \mathrm{de} \mathrm{N}$. Já no Cambissolo, constatou-se efeito linear positivo, com produção crescente com as doses (Figura 7A). Na interação fonte $\mathrm{x}$ dose, verificou-se efeito linear positivo nas fontes amoniacais, que produziram as maiores médias de PMST com o aumento das doses, sendo 6,88 g quando aplicado o sulfato de amônio e 4,89 g com a aplicação do nitrato de amônio. Já no nitrato de cálcio o ponto de máxima produção de matéria seca total $(2,68 \mathrm{~g})$ foi obtido na dose de $138 \mathrm{mg} / \mathrm{dm}^{3}$, sendo quase três vezes menor que a produção com o sulfato de amônio (Figura 7B).
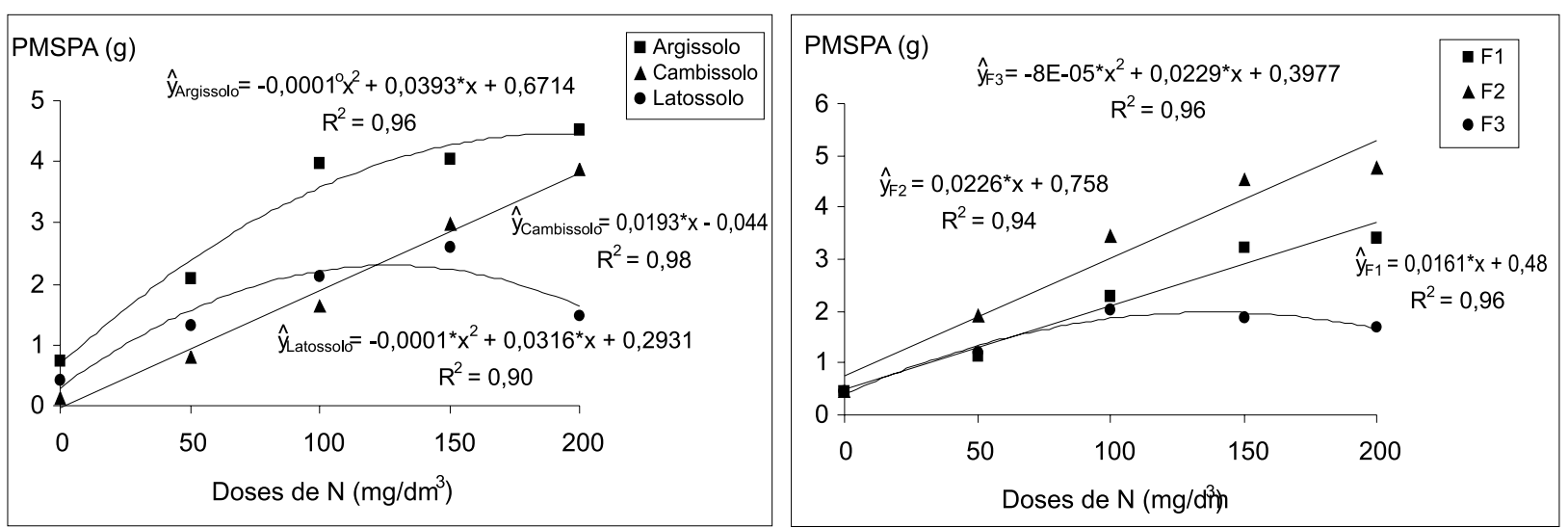

Figura 4A - Peso de matéria seca da parte aérea (PMSPA) de mudas de jacarandá-da-bahia em resposta às doses de N aplicadas, nos três solos estudados. $* \mathrm{e}^{\circ}$ coeficientes significativos a 5 e $10 \%$, respectivamente.

Figura 4B - Peso de matéria seca da parte aérea (PMSPA) de mudas de jacarandá-da-bahia em resposta às doses de N aplicadas, para as três fontes nitrogenadas ( $\mathrm{F} 1=$ nitrato de amônio; F2=sulfato de amônio e F3=nitrato de cálcio). * coeficientes significativos a $5 \%$.

Figure 4A - Shoot dry weight (PMSPA) of jacarandá-da-Bahia seedlings as a function of $N$ doses, of the three studied soils. * and ${ }^{\circ}$, significant coefficients at 5 and $10 \%$, respectively.

Figure 4B - Shoot dry weight (PMSPA) of jacarandá-da-Bahia seedlings as a function of $N$ doses, for three nitrogen sources $(F 1=$ ammonium nitrate; $F 2=$ ammonium sulfate and $F 3=$ calcium nitrate $)$ * significant coefficients at $5 \%$. 

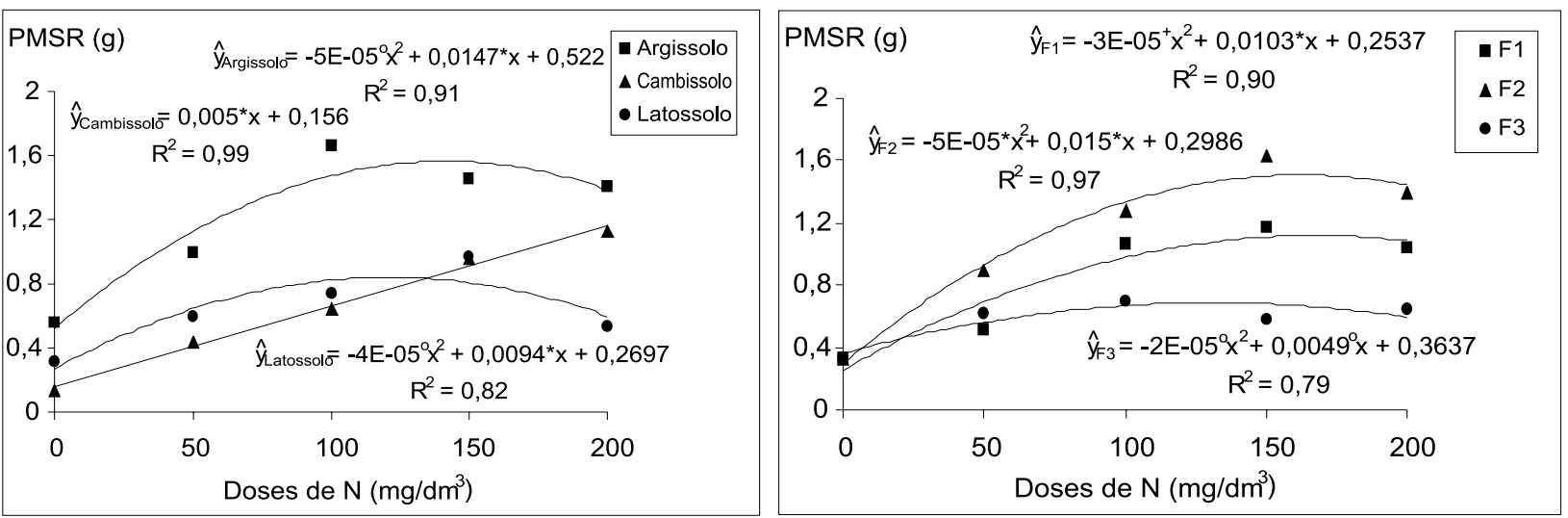

Figura 5A - Peso de matéria seca das raízes (PMSR) de mudas de jacarandá-da-bahia em resposta às doses de $\mathrm{N}$ aplicadas, nos três solos estudados. ${ }^{*} \mathrm{e}^{\circ}$ coeficientes significativos a 5 e $10 \%$, respectivamente.

Figura 5B - Peso de matéria seca das raízes (PMSR) de mudas de jacarandá-da-bahia em resposta às doses de N aplicadas, nas três fontes nitrogenadas $(\mathrm{F} 1=$ nitrato de amônio; $\mathrm{F} 2=$ sulfato de amônio e F3 = nitrato de cálcio). * o $\mathrm{e}^{+}$coeficientes significativos a $5,10 \%$ e $15 \%$, respectivamente.

Figure 5A - Roots dry weight (PMSR) of jacarandá-of-bahia seedlings as a function of $N$ doses, for three studied soils. $*$ and ${ }^{\circ}$, significant coefficients at 5 and $10 \%$, respectively.

Figure 5B-Roots dry weight (PMSR) of jacarandá-da-bahia seedlings as a function of $N$ doses, for three nitrogen sources $(F 1=$ ammonium nitrate; $F 2=$ ammonium sulfate and $F 3=$ calcium nitrate $) . *^{*}{ }^{o}$ and ${ }^{+}$, significant coefficients at $5,10 \%$ and $15 \%$, respectively.

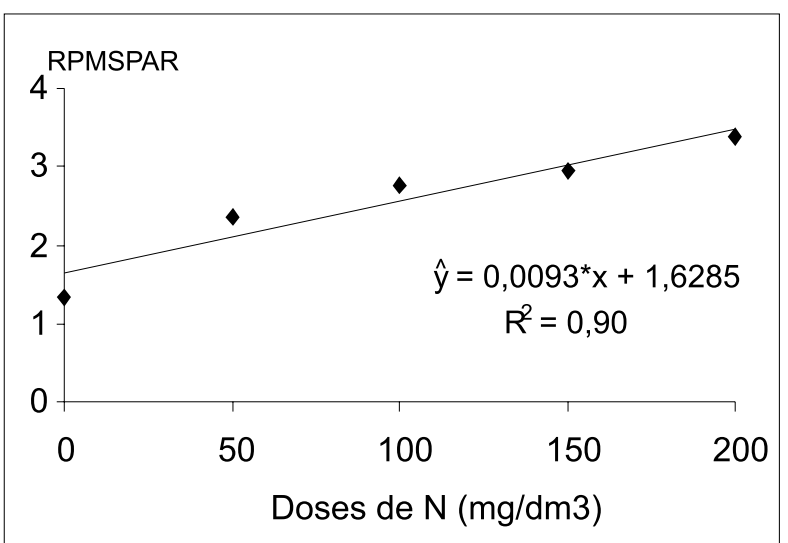

Figura 6 - Relação peso de matéria seca da parte aérea/peso de matéria seca das raízes (RPMSPAR) de mudas de jacarandá-da-bahia em resposta às doses de nitrogênio aplicadas. * coeficientes significativos a $5 \%$.

Figure 6-Shoot dry weight/root ratio dry weight (RPMSPAR) of jacarandá-da-bahia seedlings, as a function of $N$ doses. * significant coefficients at $5 \%$.

Sobre a relação altura da parte aérea/peso de matéria seca da parte aérea (RHPMSPA), apenas a interação solo $\mathrm{x}$ dose foi significativa, verificando-se efeito quadrático sobre essa característica em todos os solos.
Os melhores valores dessa característica foram 11,94 para Argissolo Vermelho-Amarelo, 16,17 para Cambissolo e 29,30 para Latossolo Vermelho-Amarelo, obtidos nas doses de 141,151 e $117 \mathrm{mg} / \mathrm{dm}^{3}$ de $\mathrm{N}$, respectivamente (Figura 8), independentemente da fonte aplicada. Essa relação não é comumente usada, porém pode ser de grande auxílio para predizer o potencial de sobrevivência da muda no campo. Segundo Gomes (2001), quanto menor for o quociente obtido, mais lignificada será a muda e maior a capacidade de sobrevivência no campo, de modo que, neste trabalho, os resultados indicaramm que o Argissolo Vermelho-Amarelo mostrou-se superior aos demais.

Verificou-se no Índice de Qualidade de Dikson (IQD) que a interação fonte x dose foi significativa, com efeito quadrático sobre esse índice nas fontes amoniacais, que produziram os maiores índices $(0,41$ e 0,34 nas doses de 185 e $200 \mathrm{mg} / \mathrm{dm}^{3}$ para sulfato de amônio e nitrato de amônio, respectivamente); logo, mudas de melhor qualidade, quando comparadas com a fonte nítrica, que proporcionou o melhor índice $(0,20)$, na dose de $125 \mathrm{mg} / \mathrm{dm}^{3}$ de $\mathrm{N}$, porém valor inferior aos encontrados nas demais fontes (Figura 9A). O IQD é uma fórmula balanceada, em que se incluem as características morfológicas $\mathrm{H}, \mathrm{DC}$, PMST, PMSPA e 
PMSR, e, quanto maior for o valor deste índice, melhor será a qualidade da muda produzida (GOMES, 2001). Quanto aos solos, observou-se sobre o IQD efeito linear positivo para Cambissolo e efeito quadrático para Argissolo Vermelho-Amarelo e Latossolo Vermelho-Amarelo, que apresentaram o maior $(0,46)$ e o menor $(0,20)$ IQD, nas doses de 146 e $122 \mathrm{mg} / \mathrm{dm}^{3}$ de N, respectivamente (Figura
9B). Segundo Fonseca et al. (2002), o IQD foi um bom parâmetro para indicar o padrão de qualidade de mudas de Trema micrantha, crescidas nas condições de viveiro suspenso, uma vez que as mudas com maiores índices apresentaram maiores valores de diâmetro do coleto, matéria seca da parte aérea, do sistema radicular e total e menores valores da relação altura/diâmetro do coleto.
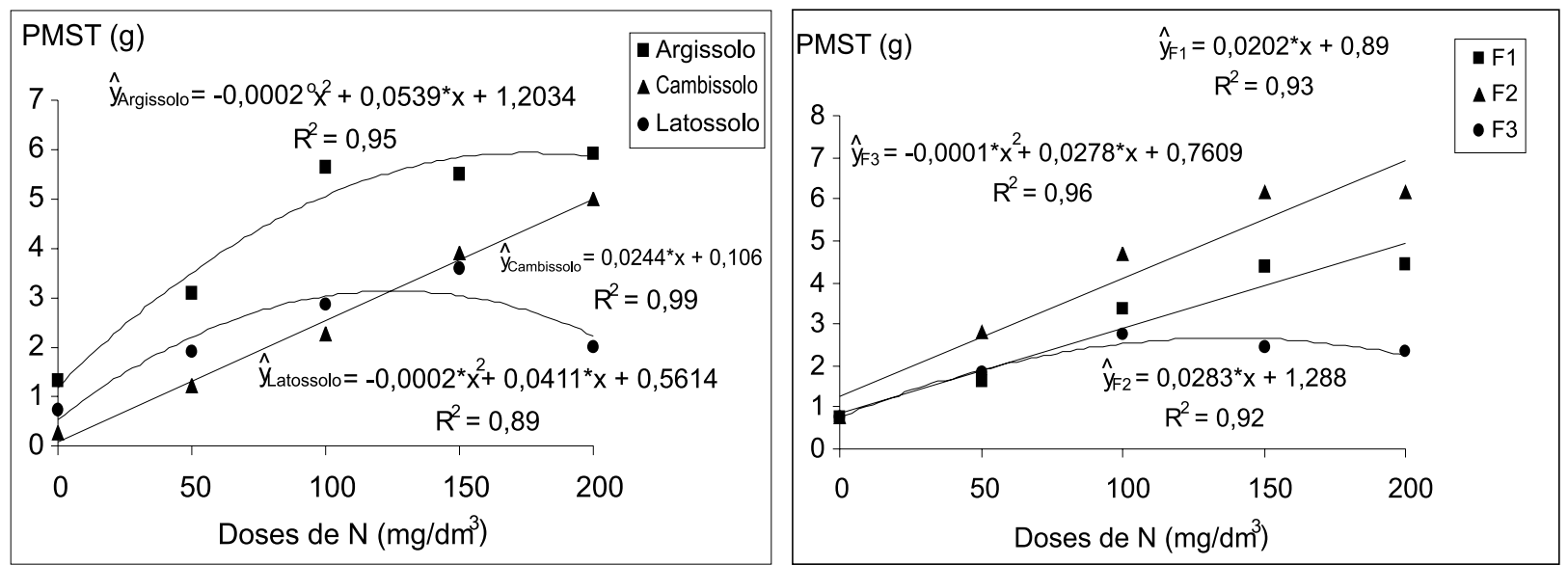

Figura 7A - Peso de matéria seca total (PMST) de mudas de jacarandá-da-bahia em resposta às doses de N aplicadas, nos três solos estudados. $* \mathrm{e}^{\circ}$ coeficientes significativos a 5 e $10 \%$, respectivamente.

Figura 7B - Peso de matéria seca total (PMST) de mudas de jacarandá-da-bahia em resposta às doses de $\mathrm{N}$ aplicadas, nas três fontes nitrogenadas $(\mathrm{F} 1=$ nitrato de amônio; F2 = sulfato de amônio e F3 = nitrato de cálcio). * coeficientes significativos a $5 \%$.

Figure 7A - Total dry weight (PMST) of jacarandá-da-Bahia seedlings as a function of $N$ doses, for three studied soils. $*$ and $^{\circ}$, significant coefficients at 5 and $10 \%$, respectively.

Figure 7B-Total dry weight (PMST) of jacarandá-da-Bahia seedlings as a function of $N$ doses, for three nitrogen sources $(F 1=$ ammonium nitrate; $F 2=$ ammonium sulfate and $F 3=$ calcium nitrate). $*$ significant coefficients at $5 \%$.

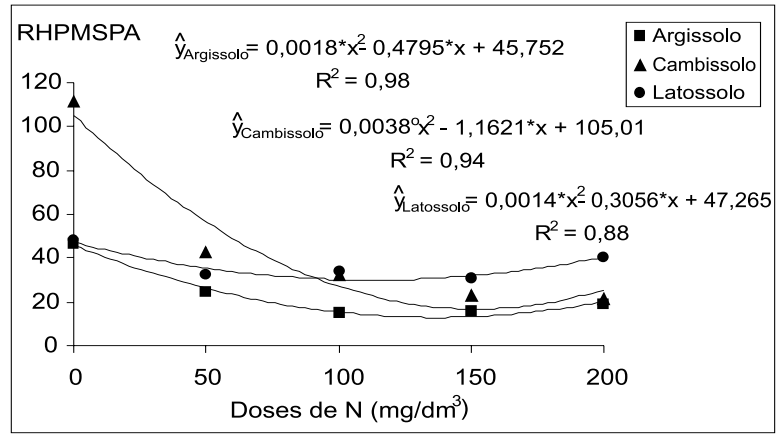

Figura 8 - Relação altura da parte aérea/peso de matéria seca da parte aérea (RHPMSPA) de mudas de jacarandá-da-bahia em resposta às doses de $\mathrm{N}$ aplicadas, nos três solos estudados. * $\mathrm{e}^{\circ}$ coeficientes significativos a 5 e $10 \%$, respectivamente.

Figure 8-Shoot height/shoot dry weight ratio (RHPMSPA) of jacarandá-da-bahia seedlings, as a function of $N$ doses, for three studied soils. * and $^{o}$, significant coefficients at 5 and $10 \%$, respectively.
Quanto ao número de nódulos radiculares, estes só foram observados no tratamento-controle e nas mudas que receberam a dose de $50 \mathrm{mg} / \mathrm{dm}^{3}$ de $\mathrm{N}$, independentemente do solo e da fonte aplicada, indicando, possivelmente, a insuficiência das menores doses para o suprimento das plantas; ao contrário das maiores, que permitiram o suprimento adequado, não justificando que as plantas gastam energia com uma associação simbiótica, se havia $\mathrm{N}$ disponível. Segundo Neto et al. (1998), em se tratando de leguminosas arbóreas que são encontradas em associação simbiótica com Rhizobium, estas podem estar sujeitas ao efeito da adição de $\mathrm{N}$ no processo de formação das mudas, bem como no local onde serão introduzidas. Adicionalmente, o nitrogênio pode afetar o processo de formação e desenvolvimento dos nódulos e, conseqüentemente, diminuir o potencial de fixação biológica de nitrogênio. 

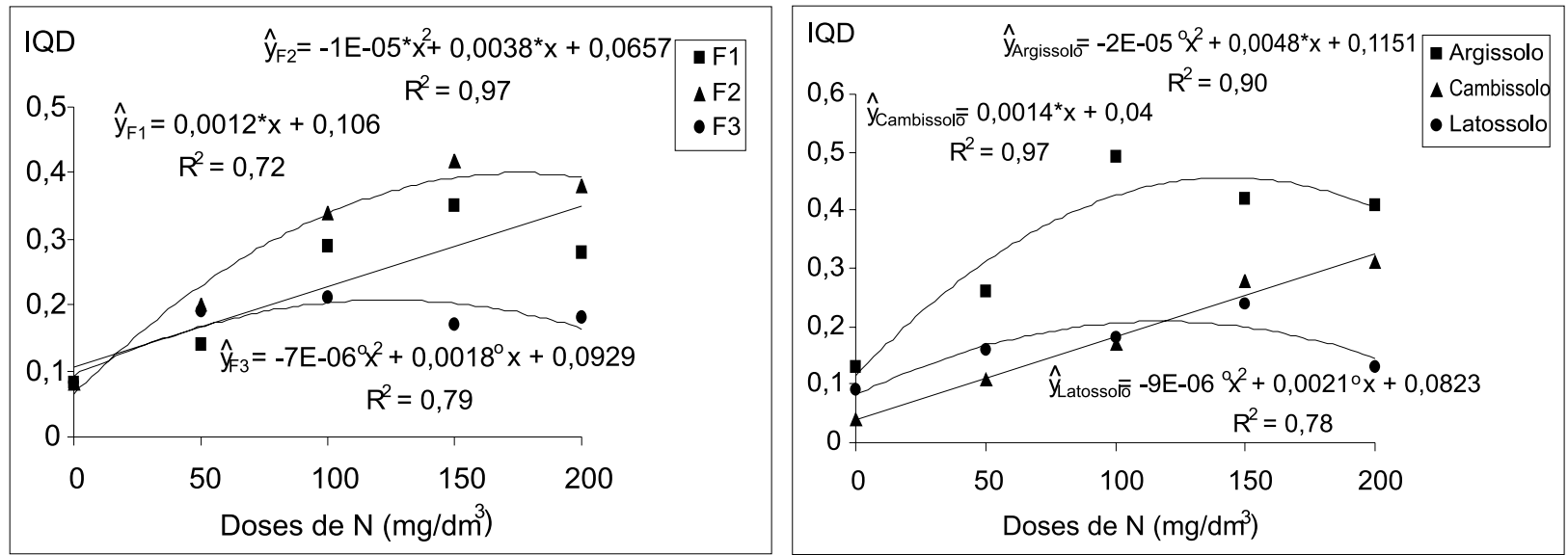

Figura 9A - Índice de qualidade de Dickson (IQD) de mudas de jacarandá-da-bahia em resposta às doses de N aplicadas, nas três fontes nitrogenadas (F1= nitrato de amônio; F2 = sulfato de amônio e F3 = nitrato de cálcio). * e o coeficientes significativos a 5 e $10 \%$, respectivamente.

Figura 9B - Índice de qualidade de Dickson (IQD) de mudas de jacarandá-da-bahia em resposta às doses de $\mathrm{N}$ aplicadas, nos três solos estudados. $* \mathrm{e}^{\circ}$ coeficientes significativos a 5 e $10 \%$, respectivamente.

Figure $9 A$ - Quality Index of Dickson (QID) of jacarandá-da-bahia seedlings as a function of doses, for three nitrogen sources $(F 1=$ ammonium nitrate; $F 2=$ ammonium sulfate and $F 3=$ calcium nitrate $) . *$ and $^{\circ}$, significant coefficients at 5 and $10 \%$, respectively.

Figure $9 B$ - Quality Index of Dickson (QID) of jacarandá-da-bahia seedlings as a function of $N$ doses, for three studied soils. $*$ and $^{\circ}$, significant coefficients at 5 and $10 \%$, respectively.

\section{CONCLUSÕES}

As mudas de jacarandá-da-bahia responderam significativamente à adição de $\mathrm{N}$-mineral nos três solos estudados, porém, dentre os três, o argissolo apresentouse como o melhor.

Dentre as fontes nitrogenadas testadas, o sulfato de amônio foi a fonte que proporcionou os melhores resultados, apresentando efeitos positivo e significativo sobre altura da parte aérea, diâmetro do coleto, relação altura da parte aérea/diâmetro do coleto, peso de matéria seca da parte aérea, peso de matéria seca das raízes, peso de matéria seca total e índice de qualidade de Dickson.

As doses utilizadas indicaram que as características avaliadas não estabeleceram sua eficiência máxima em uma única dose, mas a maioria das características teve melhor desenvolvimento na dose de $180 \mathrm{mg} / \mathrm{dm}^{3} \mathrm{de}$ $\mathrm{N}$, recomendando-se essa dose na produção de mudas de jacarandá-da-bahia, tendo como fonte o sulfato de amônio e Argissolo Vermelho-Amarelo como substrato.

\section{AGRADECIMENTOS}

Os autores agradecem à CAPES, pela concessão de bolsa de estudos; ao CNPq pela concessão de bolsas de produtividade em pesquisa, e ao Projeto PRODETAB 130-02/01, pelo financiamento do presente trabalho.

\section{REFERÊNCIAS BIBLIOGRÁFICAS}

ALVAREZ V., V. H. Equilíbrio de formas disponíveis de fósforo e enxofre em dois Latossolos de Minas Gerais, 1974. 125 f. Dissertação (Mestrado em Solos e Nutrição de Plantas) - Universidade Federal de Viçosa, Viçosa, MG, 1974.

BARROS, N. F. et al. Efeitos de recipientes na sobrevivência e no crescimento de mudas de Eucalyptus grandis no viveiro e no campo. Revista Árvore, v. 2, n. 2, p. 141-151, 1978.

BLEVINS, D. G. An overview of nitrogen metabolism in higher plants. In: POULTON, J. E.; ROMEO, J. T.; CONN, E. E. (Eds.). Plant nitrogen metabolism. New York: Plenum Press, 1989. p.234-256.

BOYER, J. N.; SOUTH, D. B. Excessive seedling height, high shoot-to-root ratio and benomyl root dip reduce survival of stored loblolly pine seedlings. Tree Planter's Notes, v.38, n.4, p. 19-22, 1987.

R. Árvore, Viçosa-MG, v.30, n.5, p.725-735, 2006 
BRISSETTE, J. C. Summary of discussion about seedling quality. In: SOUTHERN NURSERY CONFERENCES, 1984. Alexandria.

Proceedings... New Orleans: USDA. Forest Service, Southern Forest Experiment Station, 1984. p. 127-128.

CARNEIRO, J. G. A. Variações na metodologia de produção de mudas florestais afetam os parâmetros morfofisiológicos que afetam sua qualidade. Série Técnica FUPEF, n. 12, p. $1-40,1983$.

CARneiro, J. G. A. Produção e controle de qualidade de mudas florestais. Curitiba: Universidade Federal do Paraná, 1995. 451 p.

CRUZ, C. A. F. et al. Efeito de diferentes níveis de saturação por bases no desenvolvimento e qualidade de mudas de ipê-roxo (Tabebuia impetiginosa (Mart.) Standley). Scientia Forestalis, v. 21, n. 66, p. 100-107, 2004.

DANIEL, O. et al. Aplicação de fósforo em mudas de Acácia mangium Willd. Revista Árvore, v. 21, n. 2, p. 163-168, 1997.

EUCLYDES, R. F. Manual de utilização do programa SAEG (Sistema para analises estatísticas e genéticas). Viçosa, MG:

Universidade Federal de Viçosa, 1997. 59 p.

FONSECA, E. P. et al. Padrão de qualidade de mudas de Trema micrantha (L.) Blume, produzidas sob diferentes períodos de sombreamento.

Revista Árvore, v. 26, n. 4, p. 515-523, 2002.

GOMES, J. M. Parâmetros morfológicos na avaliação da qualidade de mudas de Eucalyptus grandis, produzidas em diferentes tamanhos de tubete e de dosagens de N-P-K. 2001. 126 f. Tese (Doutorado em Ciência Florestal) - Universidade Federal de Viçosa, Viçosa, MG, 2001.

GONÇALVES, J. L. M. Características do sistema radicular de Eucalyptus grandis sob diferentes condições edáficas (I Distribuição de raízes nas camadas de solo). In: CONGRESSO BRASILEIRO DE CIENCIAS DO SOLO, 21., 1995, Viçosa. Anais... Viçosa, MG: Sociedade Brasileira de Ciência do Solo, 1995. p. 876-878.

R. Árvore, Viçosa-MG, v.30, n.5, p.725-735, 2006
GONÇALVES, J. L. M. et al. Reflexos do cultivo mínimo e intensivo do solo em sua fertilidade e na nutrição das árvores. In: GONÇALVES, J. L.; BENEDETTI, V. (Eds.). Nutrição e fertilização florestal. Piracicaba: IPEF, 2000. p. 3-57.

KRAJINA, V. J.; MADOC-JONES, S.; MELLOR, G. Short communication. Ammonium and nitrate in the nitrogen economy of some conifers growing in Douglas-fir communities of the Pacific North-West of America. Soil Biology Biochemistry, v. 5, n. 1, p. 143-147, 1973.

LOCATELLI, M. et al. Efeito de fontes e doses de nitrogênio no crescimento de mudas de eucalipto. Revista Árvore, v. 8, n. 1, p. 39-52, 1984.

LORENZI, H. Árvores brasileiras: manual de identificação e cultivo de plantas arbóreas nativas do Brasil. 2. ed. Nova Odessa: Plantarum, 1998. v. 1. 368 p.

MELO, J. T. et al. Coleta, propagação e desenvolvimento inicial de espécies de cerrado. In: SANO, S. M.; ALMEIDA, S. P. (Eds.) Cerrado: ambiente e flora. Planaltina-DF: Empresa Brasileira de Pesquisa Agropecuária, $1998.556 \mathrm{p}$.

MOREIRA, F. M. S.; MOREIRA, F. W. Característica de germinação de 64 espécies de leguminosas florestais nativas da Amazônia, em condições de viveiro. Acta Amazônica, v. 26, n. 1/2, p. 3-16, 1996.

NETO, J. J.; GOI, S. R.; SPRENT, J. I. Efeito de diferentes formas de nitrogênio na nodulação e crescimento de Acacia mangium. Floresta e Ambiente, v. 5, n. 1, p. 104-110, 1998.

PASSOS, M. A. A. Efeito da calagem e de fósforo no crescimento inicial da algaroba (Prosopis juliflora (SW) DC). 1994. 57 f. Tese (Doutorado em Fitotecnia) Universidade Federal de Viçosa, Viçosa, MG, 1994. 
PARVIAINEN, J. V. Qualidade e avaliação de mudas florestais. In: SEMINÁRIO DE

SEMENTES E VIVEIROS FLORESTAIS, 1., 1981. Curitiba. Anais... Curitiba: FUPEF, 1981. p. 59-90.

SCHUMACHER, M. V. et al. Influência de diferentes doses de fósforo no crescimento de mudas de angico vermelho (Parapiptadenia rígida (Bentham). Brenan). Revista Árvore, v. 28 , n. 1, p. 149-155, 2004.
RESENDE, M.; CURI, N.; SANTANA, D. P. Pedologia e fertilidade do solo: interações e aplicações. Brasília:Ministério da Educação; Lavras:ESAL;

Piracicaba:POTAFOS, 1988. 81 p.

STURION, J. A.; ANTUNES, B. M. A. Produção de mudas de espécies florestais. In: GALVÃO, A. P. M. Reflorestamneto de propriedades rurais para fins produtivos e ambientais. Colombo: 2000. p. 125-150. 